\title{
Additional studies on triamcinolone acetonide use and misuse in sports: elimination profile after intranasal and high-dose intramuscular administrations
}

Sergi Coll ${ }^{1,2}$ (ORCID: 0000-0003-0729-4470), Núria Monfort ${ }^{1}$, Élida Alechaga ${ }^{1}$ (ORCID: 0000-0002-6698-7544), Xavier Matabosch ${ }^{1}$, Clara Pérez-Mañáa ${ }^{3,4,5}$, Rosa Ventura $^{1,2}$ (ORCID: 0000-0002-1413-8890)

(1) IMIM (Hospital del Mar Medical Research Institute), Doping Control Research Group, Catalonian Antidoping Laboratory, Barcelona, Spain

(2) Department of Experimental and Health Sciences, Universitat Pompeu Fabra, Barcelona, Spain

(3) Hospital Universitari Germans Trias i Pujol (HUGTP-IGTP), Department of Clinical Pharmacology, Badalona, Spain

(4) Department of Pharmacology, Therapeutics and Toxicology, Autonomous University of Barcelona, Cerdanyola del Vallès, Spain

(5) IMIM (Hospital del Mar Medical Research Institute), Human Pharmacology and Clinical Neurosciences Research Group, Barcelona, Spain

Corresponding author: Rosa Ventura Alemany

Doctor Aiguader 88, 08003 Barcelona, Spain

E-mail: rventura@imim.es

Phone: +0034-933160471

Fax: +0034-933160499 
Abstract

Triamcinolone acetonide (TA) is a glucocorticoid (GC) widely used in sports medicine. GCs are prohibited in sports competitions by oral, intramuscular (IM), intravenous and rectal administrations, and they are allowed by other routes considered of local action such as intranasal administration (INT). We examined the urinary profiles of TA and its metabolites after INT and high-dose IM administrations. We also measured concentrations of TA and cortisol (CORT) in plasma following IM administration.

TA was administered to healthy volunteers using INT route $(220 \mu \mathrm{g} /$ day for 3 days, $\mathrm{n}=$ 4 males and 4 females) or IM route (single dose of $40 \mathrm{mg}, \mathrm{n}=4$ males and 4 females and single dose $80 \mathrm{mg}, \mathrm{n}=4$ males). Urine and plasma samples were collected before and after administration at different time periods, and were analysed by liquid chromatography-tandem mass spectrometry.

TA concentrations in urine were constant during 23 days after IM injection (range 1.4$129.0 \mathrm{ng} / \mathrm{mL}$ ), and were very low after INT administration (range 0.0-3.5 ng/mL). For $6 \beta$-hydroxy-triamcinolone, the main TA metabolite, higher concentrations were detected (0.0-93.7 ng/mL and 15.7-973.9 ng/mL after INT and IM administrations, respectively). On the other hand, TA was detected in all plasma samples collected during 23 days after IM administration (range 0.2-5.7 ng/mL). CORT levels were largely suppressed after IM injection, and were recovered in a dose-dependent manner.

In view of the results obtained, we propose a reporting level of $5 \mathrm{ng} / \mathrm{mL}$ for TA to distinguish forbidden from allowed TA administrations in sports. We also suggest that other GCs with faster urinary elimination from the body should be considered for IM therapies in out-of-competition rather than TA, in order to reduce the possibility of reporting false adverse analytical findings. 


\section{Introduction}

Triamcinolone acetonide (TA) is a potent glucocorticoid (GC) widely used in sports medicine to treat rhinitis and acute sport injuries [1-4]. GCs are controlled in sports because of evidences of positive effects on exercise performance and health risks associated to their use due to the inhibition of the hypothalamic-pituitary-adrenal (HPA) axis [4-8]. In the 2019 World Anti-Doping Agency (WADA) Prohibited List [9], GCs are prohibited in sports competitions when administered by systemic routes (e.g., intramuscular (IM) administration), and they are allowed using other administration routes considered of local action (intranasal (INT), topical (TOP), and intra-articular (IA) administrations, among others). In an attempt to discriminate allowed from forbidden administrations, WADA established a reporting level of $30 \mathrm{ng} / \mathrm{mL}$. This criterion is based on general considerations and not on pharmacokinetic/pharmacodynamic studies of the different GCs after different administration routes, and it has shown to be not suitable for some GCs [10-18]. The current WADA criterion was evaluated by our group to detect TA misuse in sports [12, 13]. First, the metabolism of TA was studied, and 6 important metabolites were identified [12] (Figure 1S, supplementary information). Then, the urinary profiles of TA and its metabolites were evaluated after allowed administrations (TOP and INT) and prohibited IM injection at low-dose [13], and it was demonstrated that using the reporting level of $30 \mathrm{ng} / \mathrm{mL}$ proposed in WADA regulations, the use of IM injection of TA at $20 \mathrm{mg}$ is not detected. Based on these results, a reporting level of $5 \mathrm{ng} / \mathrm{mL}$ for TA was suggested to detect low-dose IM administration of TA in sports [13]. Up to our knowledge, there is not data available about the metabolic profile of TA after high-dose IM injections, and therefore, neither the current nor the proposed criteria have been evaluated to detect these treatments, which are widely prescribed to treat sport injuries. 
Moreover, it is important to the study the metabolism of TA in female subjects in order to evaluate potential gender differences.

On the other hand, a major difficulty to find a suitable discrimination criterion is the similarity in urinary profiles observed after prohibited IM and allowed IA administrations of some GCs [15-17], making the discrimination of these administration routes difficult. In a recent study performed with triamcinolone hexacetonide (THA) [15], a prodrug of TA, very high TA concentrations were detected in urine up to day 4 after a single IA administration of THA. For this reason, the evaluation of the possibility of discriminating IM from IA administrations using other matrices, such as plasma, would be of interest.

The objective of the present work was to provide more data about the metabolism of TA to improve the detection of its misuse in sports. For that purpose, the metabolic profile of TA was studied in urine after allowed (INT) and forbidden (single IM doses of 40 or $80 \mathrm{mg}$ ) administrations. Concentrations of TA and cortisol (CORT) were also measured in plasma after IM administration. 


\section{Experimental}

\subsection{Clinical studies}

Two clinical studies that involved INT and IM administrations of TA to healthy Caucasian subjects, and collection of urine and blood samples, were performed (Table 1) (protocol code IMIMFTCL/DACORSIN/2). Ethical approval of the studies were granted by the local Research Ethical Committee (Comitè Ètic d'Investigació Clínica CEIC-Parc de Salut Mar, Barcelona, Spain) and the Spanish Medicines Agency (EudraCT protocol number 2014-002862-54).

A total of twenty healthy volunteers, eight females and twelve males, were recruited (Table 2). All participants gave their written informed consent prior inclusion and underwent a general physical examination, routine laboratory test, urinalysis, and a 12lead electrocardiogram with results within normal values. All volunteers declared not to consume drugs that could alter GCs metabolism such as steroids, prohormones, contraceptives or diuretics.

In the first clinical study, four male and four female subjects received an INT treatment that consisted of daily doses of $220 \mu \mathrm{g}$ (two sprays of $55 \mu \mathrm{g}$ in each nostril) (Nasacort, Sanofi-Aventis S.A., Spain) for three consecutive days (Table 1). Urine samples were collected before administration and after administration from 0-6 h, 6-12 h and 12-24 h on days 1, 2 and 3, and from $0-12 \mathrm{~h}$ and 12-24 h on day 4 . In the second clinical study, a single IM dose (Trigon Depot®, Bristol-Myers Squibb, Spain) was administered at 40 $\mathrm{mg}$ to four males and four females, and at $80 \mathrm{mg}$ to four males (Table 1). IM administrations were gluteal injections and the urine collection was as follow: basal urine and post-administration urines from $0-6 \mathrm{~h}, 6-12 \mathrm{~h}$ and $12-24 \mathrm{~h}$ on day 1 , from $0-12$ 
$\mathrm{h}, 12-24 \mathrm{~h}$ on day 2 , and from 0-24 h on days $3,4,5$ and 6 . Additional 0-24 $\mathrm{h}$ and morning spot urines were also collected (Table 1). Urine samples were collected in sterile containers. Preliminary measurements, including specific gravity (SG) and $\mathrm{pH}$, were performed. SG was determined using an Atago UG- $\alpha$ Refractometer (Atago, Tokyo, Japan), and pH was measured by a Crison GLP $21 \mathrm{pH}$ meter (Crison, Alella, Spain). Urine samples were stored at $-20^{\circ} \mathrm{C}$ until further analysis.

Blood samples were also collected in the second clinical study (Table 1). Blood samples were drawn just before administration, $0.5,1,1.5,2,3,4$ and $6 \mathrm{~h}$ after injection and on days $1,2,3,4,9,14,18$ and 23. All blood samples were collected in the morning in order to reduce the effect of circadian rhythm in CORT measurements [19]. Blood samples were collected with $\mathrm{K}_{2}$ EDTA tubes (BD Vacutainer® spray-coated $\mathrm{K}_{2}$ EDTA Tube) according to the WADA blood collection guidelines [20]. Plasma was obtained by centrifugation (10 minutes, $3000 \mathrm{rpm}, 4^{\circ} \mathrm{C}$ ), and was immediately transferred to lowbinding tubes and stored at $-20^{\circ} \mathrm{C}$ until analysis.

\subsection{Chemical and Reagents}

Triamcinolone acetonide (TA), triamcinolone (T), cortisol (CORT), cortisol-9,11,12,12d4 (CORT-d4) and ammonium formate were obtained from Sigma (ST. Louis, MO,

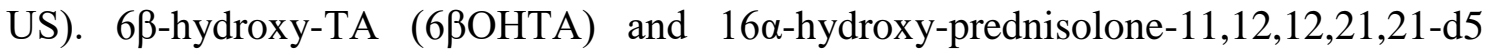
(16aOHPRED-d5) were purchased from Toronto Research Chemicals (Toronto, Canada). Triamcinolone-6-d1 acetonide-d6 (TA-d7) was obtained from CDN Isotopes (Pointe-Claire, Canada). The $\beta$-glucuronidase preparation (type Escherichia coli K12) was purchased from Roche Diagnostics, GmbH (Manheim, Germany). Analytical grade disodium hydrogen phosphate, sodium hydrogen phosphate, potassium carbonate, ethyl acetate, acetonitrile and methanol (liquid chromatography gradient grade), and formic 
acid (liquid chromatrography-mass spectrometry grade) were obtained from Merck (Darmstadt, Germany). Milli-Q water was obtained using a Milli-Q purification system (Millipore Ibérica, Barcelona, Spain). Human blank plasma was provided by Hospital del Mar (Barcelona, Spain).

\subsection{Sample Treatment}

\subsubsection{Urine samples}

The analysis of urine samples was performed using an extraction protocol previously described [13]. Briefly, two internal standards (ISTDs) (40 ng of TA-d7 and $80 \mathrm{ng}$ of $16 \alpha \mathrm{OHPRED}-\mathrm{d} 5)$ were added to $5 \mathrm{~mL}$ of urine, followed by the addition of $1.5 \mathrm{~mL} 1 \mathrm{M}$

phosphate buffer, $\mathrm{pH}$ 7. Then, enzymatic hydrolysis was performed by adding $50 \mu \mathrm{L}$ of $\beta$-glucuronidase enzyme from Escherichia coli and incubating for $1 \mathrm{~h}$ in a $55^{\circ} \mathrm{C}$ water bath. The mixture was alkalinized with $400 \mu \mathrm{L}$ of $25 \%$ potassium carbonate solution to $\mathrm{pH} 8-9$, and the steroids were extracted twice with $4 \mathrm{~mL}$ of ethyl acetate. After centrifugation ( 5 minutes at $1400 \mathrm{~g}$ ), the organic layer was transferred into a new tube and evaporated to dryness under a nitrogen stream in a water bath at $40^{\circ} \mathrm{C}$. The residue was reconstituted into $100 \mu \mathrm{L}$ of a mixture of water to acetonitrile $(3: 1, \mathrm{v} / \mathrm{v})$.

\subsubsection{Plasma samples}

For the analysis of plasma samples an extraction protocol previously published was used [15]. Two ISTDs (50 $\mu \mathrm{g}$ of CORT-d4 and $2.5 \mu \mathrm{g}$ of TA-d7) were added to $1 \mathrm{~mL}$ of plasma, followed by the addition of $1 \mathrm{~mL}$ of acetonitrile in order to precipitate proteins. Then, steroids were extracted with $5 \mathrm{~mL}$ of tert-butyl-methyl ether, and after centrifugation (10 min at $1400 \mathrm{~g}$ ), the organic layer was transferred into a new tube and 
evaporated to dryness under a nitrogen stream in a water bath at $40^{\circ} \mathrm{C}$. The residue was reconstituted into $150 \mu \mathrm{L}$ of a mixture of water to acetonitrile $(1: 1, \mathrm{v} / \mathrm{v})$.

\subsection{LC-MS/MS analyses}

LC-MS/MS analyses were carried out using a triple quadrupole (Xevo TQ MS) mass spectrometer provided with an orthogonal Z-spray-electrosptray interface (Waters Associates, Mildford, MA, USA) coupled to an Acquity ultraperformance liquid chromatographic (UPLC) system (Waters Associates) for the chromatographic separation. Nitrogen and argon (both with purity $\geq 99.999 \%$ ) were used as desolvation gas and as collision gas, respectively. The desolvation gas flow was set to approximately $1200 \mathrm{~L} / \mathrm{h}$ and the cone gas flow to $50 \mathrm{~L} / \mathrm{h}$. The nitrogen desolvation temperature was set to $450^{\circ} \mathrm{C}$ and the source temperature to $120^{\circ} \mathrm{C}$. Capillary voltage of $3.5 \mathrm{kV}$ was used in positive ionization mode.

Liquid chromatographic separations were performed using an Acquity BEH C18 column (100 x $2.1 \mathrm{~mm}$ internal diameter, $1.7 \mu \mathrm{m}$ particle size) (Waters Associates) using a column temperature of $55^{\circ} \mathrm{C}$. The mobile phase solvents $\mathrm{A}$ and $\mathrm{B}$ were water and methanol, respectively, both with $1 \mathrm{mM}$ ammonium formate and formic acid $(0.01 \%)$. For the analysis of urine samples, the percentage of organic solvent was linearly changed as follows: $0 \mathrm{~min}, 28 \% \mathrm{~B} ; 0.5 \mathrm{~min}, 28 \% \mathrm{~B} ; 6 \mathrm{~min}, 40 \% \mathrm{~B} ; 20 \mathrm{~min}$, $44 \%$ B; $20.5 \min , 90 \%$ B; $21 \min , 90 \%$ B; $21.1 \mathrm{~min}, 28 \%$ B; and $23 \mathrm{~min}, 28 \% \mathrm{~B}$ at a flow rate of $0.3 \mathrm{~mL} / \mathrm{min}$ [13]. For the analysis of plasma samples, the percentage of organic solvent was linearly changed as follows: $0 \mathrm{~min}, 30 \% \mathrm{~B} ; 0.5 \mathrm{~min}, 30 \% \mathrm{~B} ; 3 \mathrm{~min}$, $43 \% \mathrm{~B} ; 12.5 \min , 48 \% \mathrm{~B} ; 13.5 \min , 65 \% \mathrm{~B} ; 16.5 \min 95 \% \mathrm{~B} ; 16.6 \min 30 \% \mathrm{~B}$; and $18.5 \mathrm{~min}, 30 \% \mathrm{~B}$ at a flow rate of $0.3 \mathrm{~mL} / \mathrm{min}$ [15]. The injection volume in both chromatographic methods was $10 \mu \mathrm{L}$. 
In total, TA and six metabolites previously described [12, 13] (Figure 1S, Supplementary Information) were measured in urine samples, and TA and CORT were measured in plasma samples. Urine and plasma samples were analysed using MRM methods previously described [15], see Tables $1 \mathrm{~S}$ and 2S of Supplementary Information for details.

\subsection{Data analysis}

In order to correct urinary concentrations by urine dilution, the concentrations were adjusted to a reference value of 1.020 following the Levine-Fahy equation $(21,22)$ :

$$
C_{\mathrm{SG}}(n g / m L)=C(n g / m L) \times\left(1.020-1 / S G_{\text {sample }}-1\right)
$$

The libPLS software obtained from MATLAB 7.10.0(R2010a) was employed for the calculations using PCA regression [23]. Data matrix to be treated by PCA consisted of the concentrations of the 7 studied compounds as well as all the ratios between them. For the compounds that were quantitatively determined, when the concentrations found were below the limit of quantitation, half of the limit of quantitation was assigned to avoid overestimation due to increased variability in extrapolated values. For the qualitatively determined metabolites, half of the limit of detection was assigned when they were not detected to avoid NaN values in the ratios. The dimension of the matrix was 270 samples x 28 variables. Normalization pre-treatment was applied to provide similar weighs to all the 270 samples. The structure of the maps of samples and variables was investigated using the principal components (PCs) scatter plots of scores and loadings, respectively. In the distribution of samples on the PCs (plot of scores), patterns related to the type of administrations were expected to be showed. In contrast, in the distribution of variables on the PCs (plot of loadings), information regarding 
correlations and dependences of the studied markers with the administration routes were expected to be showed. 


\section{Results}

\subsection{Intranasal administration}

TA, 6ßOHTA, T and four additional metabolites (M2 to M5) were measured in all urine samples collected after INT administrations. Concentrations of TA and $6 \beta \mathrm{OHTA}$ are presented in Figure 1. As previously reported for INT administrations of TA [13], 6 $\beta$ OHTA is the main metabolite (range $0.0-93.7 \mathrm{ng} / \mathrm{mL}$ ), and TA is detected in concentrations lower than $5 \mathrm{ng} / \mathrm{mL}$ (range 0.0-3.5 ng/mL). Regarding minor metabolites, T and metabolite M2 were detected in very low amounts (ranges 0.0-1.4 $\mathrm{ng} / \mathrm{mL}$ and 0.0-1.5 arbitrary units, respectively), and metabolites M3, M4 and M5 were undetected in the majority of the samples (ranges $0.00-0.28,0.00-0.06$ and $0.00-0.03$ arbitrary units, respectively). In general, metabolites were detected in samples collected on days 1 to 3 , when volunteers were under treatment, and were almost undetectable on day 4, when there was no administration. After INT treatment, concentrations of TA and its metabolites were of the same order for males and for females.

Excretion profiles of TA and 6 3 OHTA after INT administrations are presented in Figure 2. Excretion rates showed a marked increase after each INT dose and a rapid recovery in the post-administrations hours. They linearly decreased after the last dose administered on day 3 , showing a complete elimination on day 4 . No differences were observed in excretion rates of TA and $6 \beta$ OHTA between males and females after INT administration.

\subsection{High-dose intramuscular administrations}

TA, 6 $\beta$ OHTA, $\mathrm{T}$ and metabolites $\mathrm{M} 2$ to $\mathrm{M} 5$ were measured in all urine samples collected after single IM administration at doses of 40 and $80 \mathrm{mg}$. Concentrations of TA and 6ßOHTA are presented in Figure 3 and Table 3S, and concentrations of $\mathrm{T}$ together 
with the qualitatively determined metabolites (M2 to M5) are depicted in Figure 4. All metabolites were detected up to the last sample collected at day 23 after administration. As reported for low-dose IM administration of TA [13], concentrations of all metabolites remain fairly constant during a long period of time after IM injection (i.e., 23 days), and 6ßOHTA is the main metabolite. As expected, the highest concentrations were obtained after the IM dose of $80 \mathrm{mg}$, where concentrations of TA and 6 $\beta$ OHTA ranged from 5.4 to 129.0 and from 35.8 to $973.9 \mathrm{ng} / \mathrm{mL}$, respectively. The IM dose of $40 \mathrm{mg}$ was administered to males and females, and concentrations of some metabolites were higher in males than in females, especially TA and 6ßOHTA. For TA, concentrations ranged from 2.2 to 21.3 and from 1.4 to $11.9 \mathrm{ng} / \mathrm{mL}$ for males and females, respectively; and for 6ßOHTA, concentrations ranged from 28.2 to 370.7 and from 15.7 to $184.6 \mathrm{ng} / \mathrm{mL}$ for males and females, respectively.

Since normalization of urinary concentrations by SG has been reported to reduce gender differences for other GCs [14], concentrations of TA and its metabolites were SGcorrected (Figures $2 \mathrm{~S}$ and 3S, supplementary information). However, SG-adjustment led to a reduction of the interindividual variability resulting in major gender differences.

Excretion profiles of TA and 6ßOHTA showed dose-dependent profiles, displaying an increase after administration followed by a slow elimination (Figure 2). Standard deviations of excretion rates were high indicating an elevated interindividual variability. After a single IM administration of $40 \mathrm{mg}$, excretion rates were higher in males than in females. 
The percentages of the dose of TA and $6 \beta \mathrm{OHTA}$ detected in urine during the first six days after IM administration also showed gender differences. For males, percentage values obtained after both high-doses (40 and $80 \mathrm{mg}$ ) were $0.3 \pm 0.2 \%$ for TA and $3.5 \pm 2.5 \%$ for $6 \beta$ OHTA, and for females, percentage values were lower $(0.1 \pm 0.0 \%$ for TA and $1.8 \pm 0.2 \%$ for $6 \beta$ OHTA). The values obtained for males were similar to those obtained in a previous study after IM administration of $20 \mathrm{mg}$ in males [13].

Plasma concentrations of TA and CORT were measured in all plasma samples collected after high-dose IM administrations. In Figure 5, mean plasma TA concentrations are shown together with mean CORT levels, which were expressed in percentage with respect to the basal concentration of each patient because of the high-interindividual variability in basal values. All blood samples were collected in the same time period in the morning to reduce the effect of circadian rhythm in CORT measurements [19]. Therefore, basal CORT concentrations refer to morning levels.

TA was detected in plasma up to the last sample collected on day 23 in all volunteers with concentrations ranging from 0.2 to $2.0 \mathrm{ng} / \mathrm{mL}$ and 0.5 to $5.7 \mathrm{ng} / \mathrm{mL}$ for $40 \mathrm{mg}$ and $80 \mathrm{mg}$, respectively (Figure 5). After IM administration of $40 \mathrm{mg}$, concentrations of TA in plasma obtained up to day 4 were higher in males than in females (Figure 5).

CORT levels decreased after IM injection, and the subsequent recovery to basal values took place in a dose-dependent manner (Figure 5). After IM administration of $40 \mathrm{mg}$, the CORT recovery to basal values was faster for females than males (Figure 5). CORT concentration profiles for each volunteer are presented in Figure $4 \mathrm{~S}$ of supplementary information. A decrease in plasma CORT concentrations was observed in all subjects. The earliest decrease was observed in the sample collected 30 min after administration and, for the majority of the subjects, the minimum CORT levels appeared in the sample 
collected $6 \mathrm{~h}$ post-administration. The recovery of CORT levels was dose-dependent, and different depending on the subject. After IM administration of $40 \mathrm{mg}$, CORT levels of three females (subjects 1, 2 and 3) and one male (subject 5) were recovered after 2448 h. For subjects 4, 6 and 7, CORT levels were recovered after 336-432 h, and for subject 8 , CORT concentrations were very low even $552 \mathrm{~h}$ post-injection. After IM administration of $80 \mathrm{mg}$, CORT levels remained suppressed much longer. One male participant (subject 9) did not show a total recovery until $552 \mathrm{~h}$ post-injection, and CORT levels of the other 3 males (subjects 10, 11 and 12) were low much longer, being around $30-50 \%$ of those levels obtained in basal conditions in the last sample collected $552 \mathrm{~h}$ after administration.

\subsection{Discrimination between allowed and forbidden administrations}

In order to study the best criterion to discriminate allowed from forbidden administrations of TA, a PCA was performed. Data obtained in previous studies that involved INT treatment (at the same dose as the present study) and low-dose IM injection (single dose of $20 \mathrm{mg}$ ) [13] were included in the evaluation. Therefore, in total 14 volunteers were included for INT administration ( 8 males and 6 females) and 8 male volunteers were included for IM administration of $20 \mathrm{mg}$. Since the urinary metabolites described for TA are also excreted following IA injection of THA [15], data from our previous study, where a single IA administration of THA was administered to 8 subjects [15], were also included in the PCA analysis. Data from TOP administrations obtained in our previous study was not included in the PCA analysis because TA and its metabolites were undetectable in almost all collected samples [13].

A data matrix containing the concentrations of each compound and the ratios between them was built to PCA exploration. Data included belong to urines collected up to day 
four for IM and IA administrations, and urines collected during the three days of INT treatment. The dimension of this matrix was 270 samples x 28 variables. Data were autoscaled with respect to the overall concentrations to provide similar weights to all the samples. Figure 6 shows plots of scores and biplot of PC1 vs PC2, which explained $42 \%$ of the data variance. As can be seen, in the scores plot (Figure 6a), allowed and forbidden administrations are difficult to be discriminated because IA samples are clustered together with IM samples, and some low-dose IM samples appear together with INT samples. Interestingly, IM samples were separated in a dose-dependent manner and a very acceptable discrimination was observed between INT and high-dose IM samples. While high-dose IM samples are grouped at the left side of the score plot, INT samples are located at the right side. There was not gender variation in the clustering of the samples obtained after IM injection of $40 \mathrm{mg}$ (data not shown). The biplot of PC1 vs PC2 (Figure 6b), which consists of the overlay of the scatter plots of scores and loadings, revealed those markers contributing more to the discrimination of administration routes. As can be seen, the individual concentrations of the markers correlated positively with the IM samples. This correlation is in agreement with our findings where concentrations of these markers were directly proportional to the IM dose. On the other hand, the ratios TA/M4, TA/M5, T/M4 and T/M5 correlated more strongly with the INT samples. Thus, TA, 6ßOHTA, T, metabolites M2 to M5 as well as the above mentioned ratios were further investigated.

The possibility to use TA, 6ßOHTA, T, M2, M3, M4 and M5 as markers to detect IM use was studied. Taking into account the maximum concentration values obtained after INT administrations of TA, reporting level values that ensured no false adverse analytical findings (AAFs) were proposed for each marker. In Table 3, the percentages of AAFs obtained after IM administrations using the proposed reporting levels for each 
marker are indicated. Furthermore, the current WADA criterion (reporting level of 30 $\mathrm{ng} / \mathrm{mL}$ for TA) was evaluated.

Results showed that the detection of AAFs is directly proportional to the IM dose, as expected. After $80 \mathrm{mg}$, all proposed criteria are able to detect a high percentage of AAFs except for $\mathrm{T}$ criterion. After $40 \mathrm{mg}$, the criteria proposed for TA, $6 \beta \mathrm{OHTA}, \mathrm{M} 2$ and M3 are the ones that detect the highest number of AAFs. Criteria proposed for M2 and M3 are very interesting because there were no gender differences in the detection of AAFs. After $20 \mathrm{mg}$, the highest number of AAFs were detected using a reporting level of $5 \mathrm{ng} / \mathrm{mL}$ for TA, as already reported [13]. Therefore, the best criterion to detect IM administration is a reporting level of $5 \mathrm{ng} / \mathrm{mL}$ for TA. The criterion based on $6 \beta O H T A$ did not improve the detection of IM administration at any dose compared with TA at 5 $\mathrm{ng} / \mathrm{mL}$.

In general, all proposed criteria provide very long retrospectivity due to the fact that concentrations of all metabolites remained constant during a long period of time (15 days after IM of $20 \mathrm{mg}$, and 23 days after 40 and $80 \mathrm{mg}$ ). On the other hand, it is demonstrated that the current WADA rule does not allow detection of IM administration of 20 and $40 \mathrm{mg}$, and only some samples are considered as AAF after IM administration of $80 \mathrm{mg}$ (Table 3). The ratios TA/M4, TA/M5, T/M4 and T/M5 were also assessed, but none of them showed a significant discriminatory power. 


\section{Discussion}

In this work, the urinary metabolites of TA are described following INT treatment with the maximum dosage recommended in therapeutics, and IM administration at doses of 40 and $80 \mathrm{mg}$. Moreover, the concentrations of TA and CORT in plasma were measured after IM administration.

The sample preparation procedure applied to urine samples allowed for the detection of the sum of both free and glucoronoconjugated metabolites. As expected, concentrations of TA and its metabolites were greater after IM administration than after INT therapy. After IM administration, TA and its metabolites exhibited slow elimination profiles showing constant concentration values during 23 days. This slow elimination is due to the depot formulation of the TA medication administered.

Results of the metabolic study in urine demonstrated that using the current WADA criterion to detect TA misuse (reporting level of $30 \mathrm{ng} / \mathrm{mL}$ for TA) a very low number of AAFs are detected after IM administration. In view of the results obtained (Table 3) and taking into account that metabolites M2 to M3 are not commercially available, it is confirmed the best discriminatory marker is TA with a reporting level of $5 \mathrm{ng} / \mathrm{mL}$ for TA, as previously reported [13]. However, M2 and M3 could also be suitable markers since a large number of samples are considered as AAF after IM administration and the same percentage of AAFs is detected for males and females (Table 3). For this reason, it would be interesting to quantify metabolites M2 and M3 with their corresponding standards in order to evaluate a reporting level for each marker based on concentrations. It is important to notice that the excretion of metabolites after IM administration is slow and metabolites concentrations remain constant during a very long period of time. Thus, the detection of IM administration of TA using the discrimination criteria proposed 
(Table 3) is very long, and there is a strong chance to report false AAFs from an IM administration of TA performed in an out-of-competition period close to an incompetition anti-doping test. For this reason, it is strongly recommended that other GCs with faster urinary elimination from the body should be considered for IM therapies rather than TA, in order to reduce the probability of reporting false AAFs after a long period following IM injection.

On the other hand, urinary concentrations of TA and its metabolites obtained after prohibited IM administrations are of the same order than those obtained after allowed IA administration of THA [15], and no discrimination could be performed by PCA. Therefore, it seems that using urinary markers is not possible to discriminate IM from IA administrations.

After IM administration, concentrations of TA and some metabolites were greater in males than in females. Although normalization of urinary concentrations by SG has been reported to reduce gender differences for other GCs [14], SG-adjustment did not reduce gender variation of TA metabolism. Subjects involved in the present study were healthy volunteers who were asked not to take drugs which are known that could alter GCs metabolism, such as steroids, diuretics or other drugs. Therefore, gender differences observed are not due to concurrent medications or administration of other drugs. In fact, gender differences observed are in agreement with results obtained in the evaluation of the urinary profile of THA [15], indicating that gender differences may exist in the TA metabolism.

Concentrations of TA in plasma were also measured after IM administration. TA was detected in all plasma samples collected up to day 23 after administration, at concentrations of the same order than those obtained after allowed IA administration of 
THA [15]. Thus, IM and IA administrations cannot be discriminated using plasma concentrations of TA.

It is well known that IM administration of GCs have systemic effects [8]. In this study, CORT concentrations were assessed before and after IM injection of TA, and it was observed that CORT levels were largely suppressed after administration, showing a profound impact on the HPA axis. The decrease in plasma concentrations of CORT observed after IM administration of TA is similar to the depletion of CORT levels found after IA administration of THA [15] and other GCs [24-26], showing that IM and IA administrations have similar effects on the HPA axis. 


\section{Conclusion}

The assessment of the TA metabolism in urine after INT and high-dose IM administration confirms that the current WADA criterion is not suitable to detect IM administrations. The best criterion to detect TA misuse seems to be a reporting level of $5 \mathrm{ng} / \mathrm{mL}$ for TA. However, the quantitation of metabolites M2 and M3 with their corresponding standards could also be useful to find a suitable discrimination criterion. Due to the slow elimination of TA metabolites after IM administration, false AAFs could be reported due to an IM injection performed in an out-of-competition period. For this reason, it is suggested that other GCs with faster urinary elimination from the body should be considered for IM therapy in sports.

Results obtained demonstrate important similarities between IM and IA administrations not only in metabolites concentrations found in urine and plasma, but also in the effect on the HPA axis. These findings support the argument that the status of the local injections of GCs in the WADA prohibited list should be revised. 


\section{Funding}

This work was supported by the World Antidoping Agency [grants numbers 13D22RV, and 16C11RV]; Consell Català de 1'Esport (Generalitat de Catalunya); and AGAUR (Generalitat de Catalunya) [reference number 2017SGR81]. 


\section{REFERENCES}

[1] Munk ZM, LaForce C, Furst JA, Simpson B, Feiss G, Smith JA. Efficacy and safety of triamcinolone acetonide aqueous nasal spray in patients with seasonal allergic rhinitis. Ann Allergy Asthma Immunol 1996;77(4):277-81. http://dx.doi.org/10.1016/S1081-1206(10)63320-0

[2] Levine WN, Bergfeld JA, Tessendorf W, Moorman CT. Intramuscular corticosteroid injection for hamstring injuries. A 13-year experience in the National Football League. Am J Sports Med 2000;28(3):297-300. http://dx.doi.org/10.1177/03635465000280030301

[3] Kim C, Cashdollar MR, Mendicino RW, Catanzariti AR, Fuge L. Incidence of plantar fascia ruptures following corticosteroid injection. Foot Ankle Spec 2010;3(6):335-7. http://dx.doi.org/10.1177/1938640010378530

[4] Stevens KJ, Crain JM, Akizuki KH, Beaulieu CF. Imaging and Ultrasound-Guided Steroid Injection of Internal Oblique Muscle Strains in Baseball Pitchers. Am J Sports Med 2010;38(3):581-5. http://dx.doi.org/10.1177/0363546509350105

[5] Duclos M. Evidence on ergogenic action of glucocorticoids as a doping agent risk. Physician Sportsmed 2010;38(3):121-7. http://dx.doi.org/10.3810/psm.2010.10.1817

[6] Keller-Wood ME, Dallman MF. Corticosteroid inhibition of ACTH secretion. Endocr Rev 1984;5(1):1-24. http://dx.doi.org/10.1210/edrv-5-1-1

[7] Henzen C, Suter A, Lerch E, et al. Suppression and recovery of adrenal response after short-term, high-dose glucocorticoid treatment. The Lancet 2000;355:542-45. http://dx.doi.org/10.1016/S0140-6736(99)06290-X

[8] Joseph RM, Hunter AL, Ray DW, Dixon WG. Systemic glucocorticoid therapy and adrenal insufficiency in adults: A systematic review. Semin Arthritis Rheum 2016 ;46(1):133-41. http://dx.doi.org/10.1016/j.semarthrit.2016.03.001

[9] The World Anti-Doping Code. The 2019 Prohibited List. International Standard. [World Anti-Doping Agency Website]. Available at: https://www.wadaama.org/sites/default/files/wada_2019_english_prohibited_list.pdf (Accessed March 2019).

[10] Matabosch X, Pozo OJ, Monfort N, et al. Urinary profile of methylprednisolone metabolites after oral and topical administrations. J Steroid Biochem Mol Biol 2013;138:214-21. http://dx.doi.org/10.1016/j.jsbmb.2013.05.019

[11] Matabosch X, Pozo OJ, Pérez-Mañá C, et al. Discrimination of prohibited oral use from authorized inhaled treatment of budesonide in sports. Ther Drug Monit 2013;35(1):118-28. http://dx.doi.org/10.1097/FTD.0b013e3182787b20 
[12] Matabosch X, Pozo OJ, Papaseit E, et al. Detection and characterization of triamcinolone acetonide metabolites in human urine by LC-MS/MS after intramuscular administration. Rapid Commun Mass Spectrom 2014;28(16):1829-39. http://dx.doi.org/10.1002/rcm.6965

[13] Matabosch X, Pozo OJ, Pérez-Mañá C, et al. Evaluation of the reporting level to detect triamcinolone acetonide misuse in sports. J Steroid Biochem Mol Biol 2014;145:94-102. http://dx.doi.org/10.1016/j.jsbmb.2014.09.018

[14] Coll S, Matabosch X, Papakonstatinou K, Pérez-Mañá C, Mateus JA, Ventura R. Budesonide use and misuse in sports: elimination profiles of budesonide and metabolites after intranasal, high-dose inhaled and oral administrations. Drug Test Anal 2019; Submitted.

[15] Coll S, Matabosch X, Llorente-Onaindia J, et al. Elimination profile of triamcinolone hexacetonide and its metabolites in human urine and plasma after a single intra-articular administration. Drug Test Anal 2019; http://dx.doi.org/10.1002/dta.2614

[16] Chang CW, Huang TY, Tseng YC, et al. Positive doping results caused by the single-dose local injection of triamcinolone acetonide. Forensic Sci Int 2014;244:1-6. http://dx.doi.org/10.1016/j.forsciint.2014.07.024

[17] Simoes SMS, Calçada M, Horta L, de la Torre X. Methylprednisolone detection in urine following local and oral administrations. In. Recent Advances in Doping Analysis (13). Schänzer W, Geyer H, Gotzmann A, Mareck U (Eds). Sportverlag Strauss, Cologne, Germany, 411-414 (2005).

[18] Ventura R, Matabosch X, Segura J. Bioanalytical techniques in discrimination between therapeutic and abusive use of drugs in sport. Bioanalysis 2016;8(9):965-80. http://dx.doi.org/10.4155/bio.15.253

[19] Guignard MM, Pesquies PC, Serrurier BD, Merino DB, Reinberg AE. Circadian rhythms in plasma levels of cortisol, dehydroepiandrosterone, $\Delta 4$-adrostenedione, testosterone and dihydrotestosterone of healthy young men. Acta Endoc 1980;94:53645 .

[20] WADA ISTI - Blood Sample Collection Guidelines [World Anti-Doping Agency Website]. Available at: https://www.wadaama.org/sites/default/files/resources/files/guidelines_blood_sample_collection_v5_sept _2016.pdf (Accessed March 2019).

[21] WADA Technical Document - TD2018DL [World Anti-Doping Agency Website]. Available at: https://www.wadaama.org/sites/default/files/resources/files/td2018dl_v1_en.pdf (Accessed March 2019).

[22] Levine, L., Fahy, JP. Evaluation of urinary lead determinations. J. Ind. Hyg. Toxicol 1996;20:379-391. 
[23] Li H-D, Xu Q-S, Liang Y-Z. (2014) libPLS: An Integrated Library for Partial Least Squares Regression and Discriminant Analysis. PeerJ PrePrints 2:e190v1, source codes available at www.libpls.net.

[24] Gless KH, Klee HR, Vecsei P, Weber M, Haack D, Lichtwald K. Plasma concentration and systemic effect of betamethasone after intra-articular injection. Dtscg Med Wochenschr 1981;106(22):704-07. http://dx.doi.org/10.1055/s-2008-1070385

[25] Derendorf H, Mollmann H, Gruner A, Haack D, Gyselby G. Pharmacokinetics and pharmacodynamics of glucocorticoid suspensions after intra-articular adminsitration. Clin Pharmacol Ther 1986;39(3):313-17.

[26] Weitoft T, Rönnblom L. Glucocorticoid resorption and influence on the hypothalamic-pituitary-adrenal axis after intra-articular treatment of the knee in resting and mobile patients. Ann Rheum Dis 2006;65(7):955-57. http://dx.doi.org/10.1136/ard.2005.041525 


\section{Tables}

Table 1. Clinical studies performed (see text for explanation).

\begin{tabular}{|c|c|c|c|c|c|c|}
\hline \multirow{2}{*}{ study } & \multirow{2}{*}{$\mathbf{n}$} & \multirow{2}{*}{ sex } & \multirow{2}{*}{ route } & \multirow{2}{*}{ dose/day } & \multicolumn{2}{|l|}{ sample collection $^{\mathrm{a}}$} \\
\hline & & & & & urine & blood \\
\hline 1 & $4 / 4$ & $\mathrm{M} / \mathrm{F}$ & INT & $220 \mu \mathrm{g}$ x 3 days & 24-hour total urine up to day 4 & - \\
\hline \multirow[t]{2}{*}{2} & $4 / 4$ & $\mathrm{M} / \mathrm{F}$ & IM & $40 \mathrm{mg}$ & $\begin{array}{l}\text { 24-hour total urine up to day } 6 \text {, and days } 9,14 \text { and } 23 \\
\text { spot urines on days } 7,11,16,18 \text { and } 21\end{array}$ & up to day 23 \\
\hline & 4 & M & IM & $80 \mathrm{mg}$ & $\begin{array}{l}\text { 24-hour total urine up to day } 6^{\mathrm{b}} \\
\text { spot urines on days } 7,8,9,11,14,16,18,21 \text { and } 23\end{array}$ & up to day 23 \\
\hline
\end{tabular}

a: Collection periods after administration are detailed in the text

b: For 1 volunteer, 24-hour total urine was also collected on days 9, 14 and 23 .

Table 2. Subjects' characteristics.

\begin{tabular}{|c|c|c|c|c|c|c|}
\hline study & $\operatorname{sex}$ & $\mathbf{n}$ & age (years) & height (cm) & weight (kg) & BMI $\left(\mathrm{kg} / \mathrm{m}^{2}\right)$ \\
\hline \multirow[t]{2}{*}{1} & $\mathrm{M}$ & 4 & $27 \pm 8$ & $175 \pm 14$ & $67 \pm 6$ & $22 \pm 3$ \\
\hline & $\mathrm{F}$ & 4 & $26 \pm 6$ & $162 \pm 5$ & $56 \pm 4$ & $21 \pm 2$ \\
\hline \multirow[t]{2}{*}{2} & $\mathrm{M}$ & 8 & $26 \pm 6$ & $173 \pm 9$ & $68 \pm 5$ & $23 \pm 2$ \\
\hline & $\mathrm{F}$ & 4 & $21 \pm 2$ & $159 \pm 3$ & $54 \pm 5$ & $21 \pm 2$ \\
\hline
\end{tabular}

Values are mean \pm SD. 
Table 3. Percentage of AAFs detected after IM administration of TA at different doses using different reporting levels. Reporting levels proposed ensure no false AAFs after INT administrations of TA. Data included involve results from a previous study where TA was IM administered at $20 \mathrm{mg}$ (13).

\begin{tabular}{|c|c|c|c|c|c|c|c|c|}
\hline \multicolumn{9}{|c|}{ IM $20 \mathrm{mg}, \mathrm{n}=8$ males } \\
\hline \multirow{2}{*}{ Compounds } & \multirow{2}{*}{$\begin{array}{l}\text { Reporting levels } \\
(\mathrm{ng} / \mathrm{mL})^{\mathrm{a}}\end{array}$} & \multicolumn{7}{|c|}{ AAFs (\%) } \\
\hline & & $0-8 \mathrm{~h}$ & $8-24 \mathrm{~h}$ & $24-48 \mathrm{~h}$ & $48-72 \mathrm{~h}$ & $72-96 \mathrm{~h}$ & $120-144 \mathrm{~h}$ & $192-360 \mathrm{~h}$ \\
\hline \multirow{2}{*}{$\mathrm{TA}$} & 5 & 40 & 60 & 60 & 40 & 70 & 55 & 44 \\
\hline & 30 & 0 & 0 & 0 & 0 & 0 & 0 & 0 \\
\hline 6ßОНTA & 100 & 0 & 40 & 35 & 60 & 40 & 30 & 19 \\
\hline $\mathrm{T}$ & 2 & 0 & 0 & 0 & 0 & 0 & 0 & 0 \\
\hline M2 & 2 & 0 & 20 & 20 & 20 & 20 & 10 & 0 \\
\hline M3 & 0.45 & 10 & 30 & 25 & 20 & 30 & 15 & 12 \\
\hline M4 & 0.10 & 0 & 10 & 15 & 10 & 10 & 10 & 6 \\
\hline M5 & 0.05 & 0 & 37 & 45 & 40 & 40 & 25 & 31 \\
\hline \multicolumn{9}{|c|}{ IM $40 \mathrm{mg}, \mathrm{n}=4$ females } \\
\hline \multirow{2}{*}{ Compounds } & \multirow{2}{*}{$\begin{array}{l}\text { Reporting levels } \\
(\mathrm{ng} / \mathrm{mL})^{\mathrm{a}}\end{array}$} & \multicolumn{7}{|c|}{ AAFs $(\%)$} \\
\hline & & $0-12 \mathrm{~h}$ & $12-24 \mathrm{~h}$ & $24-48 \mathrm{~h}$ & $48-72 \mathrm{~h}$ & $72-96 \mathrm{~h}$ & $120-168 \mathrm{~h}$ & $192-552 \mathrm{~h}$ \\
\hline \multirow[t]{2}{*}{ TA } & 5 & 38 & 50 & 50 & 50 & 50 & 58 & 64 \\
\hline & 30 & 0 & 0 & 0 & 0 & 0 & 0 & 0 \\
\hline $6 \beta \mathrm{OHTA}$ & 100 & 12 & 25 & 12 & 25 & 50 & 17 & 37 \\
\hline $\mathrm{T}$ & 2 & 0 & 0 & 0 & 0 & 0 & 0 & 0 \\
\hline M2 & 2 & 75 & 75 & 75 & 75 & 75 & 75 & 79 \\
\hline M3 & 0.45 & 75 & 75 & 75 & 75 & 50 & 92 & 96 \\
\hline M4 & 0.10 & 0 & 0 & 0 & 0 & 0 & 0 & 14 \\
\hline M5 & 0.05 & 13 & 25 & 13 & 0 & 25 & 25 & 54 \\
\hline \multicolumn{9}{|c|}{ IM $40 \mathrm{mg}, \mathrm{n}=4 \mathrm{males}$} \\
\hline \multirow{2}{*}{ Compounds } & \multirow{2}{*}{$\begin{array}{l}\text { Reporting levels } \\
(\mathrm{ng} / \mathrm{mL})^{\mathrm{a}}\end{array}$} & \multicolumn{7}{|c|}{ AAFs (\%) } \\
\hline & & $0-12 \mathrm{~h}$ & $12-24 \mathrm{~h}$ & $24-48 \mathrm{~h}$ & $48-72 \mathrm{~h}$ & $72-96 \mathrm{~h}$ & $120-168 \mathrm{~h}$ & $192-552 \mathrm{~h}$ \\
\hline \multirow[t]{2}{*}{ TA } & 5 & 100 & 100 & 100 & 100 & 100 & 83 & 76 \\
\hline & 30 & 0 & 0 & 0 & 0 & 0 & 0 & 0 \\
\hline 6ßОНТА & 100 & 40 & 75 & 88 & 100 & 75 & 67 & 34 \\
\hline $\mathrm{T}$ & 2 & 0 & 0 & 0 & 0 & 0 & 0 & 0 \\
\hline M2 & 2 & 100 & 100 & 100 & 100 & 100 & 100 & 100 \\
\hline M3 & 0.45 & 75 & 100 & 100 & 100 & 100 & 83 & 83 \\
\hline M4 & 0.10 & 0 & 50 & 38 & 50 & 25 & 33 & 10 \\
\hline M5 & 0.05 & 38 & 75 & 75 & 75 & 75 & 67 & 52 \\
\hline \multicolumn{9}{|c|}{ IM $80 \mathrm{mg}, \mathrm{n}=4$ males } \\
\hline \multirow{2}{*}{ Compounds } & \multirow{2}{*}{$\begin{array}{l}\text { Reporting levels } \\
(\mathrm{ng} / \mathrm{mL})^{\mathrm{a}}\end{array}$} & \multicolumn{7}{|c|}{ AAFs $(\%)$} \\
\hline & & $0-12 \mathrm{~h}$ & $12-24 \mathrm{~h}$ & $24-48 \mathrm{~h}$ & $48-72 \mathrm{~h}$ & $72-96 \mathrm{~h}$ & $120-168 \mathrm{~h}$ & $192-552 \mathrm{~h}$ \\
\hline \multirow[t]{2}{*}{ TA } & 5 & 100 & 100 & 100 & 100 & 100 & 100 & 100 \\
\hline & 30 & 38 & 25 & 50 & 25 & 25 & 33 & 10 \\
\hline $6 \beta \mathrm{OHTA}$ & 100 & 62 & 100 & 88 & 75 & 75 & 75 & 65 \\
\hline $\mathrm{T}$ & 2 & 25 & 25 & 13 & 25 & 0 & 0 & 0 \\
\hline M2 & 2 & 100 & 100 & 100 & 100 & 100 & 100 & 97 \\
\hline M3 & 0.45 & 100 & 100 & 100 & 100 & 100 & 100 & 100 \\
\hline M4 & 0.10 & 37 & 100 & 100 & 100 & 100 & 100 & 84 \\
\hline M5 & 0.05 & 63 & 100 & 100 & 100 & 100 & 100 & 100 \\
\hline
\end{tabular}

a For M2, M3, M4 and M5, reporting levels are expressed in arbitrary units. 


\section{Figures legends}

Figure 1. Concentrations of TA and $6 \beta$ OHTA after INT administrations of TA. Time (days, d) and subjects' sex (female, F; male, M) are noted. Dotted line corresponds to the general reporting level of $30 \mathrm{ng} / \mathrm{mL}$ for GCs.

Figure 2. Excretion profiles $(\mathrm{ng} / \mathrm{min}$, mean $+\mathrm{SD})$ of TA and $6 \beta \mathrm{OHTA}$ in samples collected after INT treatment or IM administrations at single doses of 40 and $80 \mathrm{mg}$. Dotted line corresponds to females and full line corresponds to males. Arrows indicate administration.

Figure 3. Concentrations of TA and $6 \beta \mathrm{OHTA}$ after IM administrations of TA at single doses of 40 and $80 \mathrm{mg}$. Time (hours, h) is noted. Dotted line corresponds to the general reporting level of $30 \mathrm{ng} / \mathrm{mL}$ for GCs

Figure 4. Amounts of T and 4 additional TA metabolites after IM administrations of TA at single doses of 40 and $80 \mathrm{mg}$. For T, concentrations $(\mathrm{ng} / \mathrm{mL})$ are shown. For M2 to M5, boxplots were constructed using relative signal to the ISTD. Time (hours, $h$ ) is noted.

Figure 5. Concentrations of TA $(\mathrm{ng} / \mathrm{mL})$ and CORT (percentage of change) as a function of time after IM administration at single doses of $40 \mathrm{mg}$ (males, circle; females, square) and $80 \mathrm{mg}$ (males, triangle).

Figure 6. Discrimination between different TA administrations by PCA. (A) Scores plot. (B) Biplot. Sample assignation: INT, asterisks; IM $20 \mathrm{mg}$, triangles; IM $40 \mathrm{mg}$, 
circles; IM $80 \mathrm{mg}$, crosses; and IA $40 \mathrm{mg}$ of THA, squares. Data included involve results from INT, IM and IA administrations performed in previous studies $(13,15)$ in addition to the results obtained in the present study. 